\title{
A method of construction of auditory stimulus patterns for use in cross-modal and intramodal matching tests
}

\author{
RONALD F. JARMAN \\ University of British Columbia, Vancouver, British Columbia, Canada V6T 1 W5
}

\begin{abstract}
Techniques for constructing auditory stimulus patterns in tests of cross-modal and intramodal matching ability are discussed. An example of a set of tests is given, and the method of construction for the auditory stimuli is described.
\end{abstract}

In a recent review, Freides (1974) discusses the use and significance of the sensory modality matching paradigm in the study of human information processing. A major difficulty in sensory modality matching research is the lack of a practical technique for generating auditory-temporal stimulus patterns. As a consequence, the parameters for test construction as suggested by Chalfant and Scheffelin (1969) have hardly begun to be utilized.

A method of construction currently in use is to generate a solid tone tape and splice portions to a second tape. The physical lengths of the spliced portions are determined by the lengths of tones and pauses desired and by the tape speed. The technique is very laborious and would require about 400 splices in the tests to be discussed here.

At least two other methods of test construction can be used. The technique with greatest potential is computer programming. Durations of tones and pauses and complexity of patterns can be controlled very effectively by a computer, making it feasible to construct many variations of a test. Another technique, which is a compromise of the two noted above, is described in this paper.

\section{TEST DESCRIPTIONS}

The method of generating auditory-temporal stimulus patterns was devised in order that four tests of sensory modality matching could be constructed. The tests consist of combinations of auditory-temporal and visualspatial stimulus patterns. ${ }^{1}$ The auditory-temporal stimuli are presented by a tape recorder, and the visualspatial stimuli are presented on slides. The patterns are given in Figure 1.

\section{Auditory-Auditory Matching}

This task involves matching a stimulus pattern of

Requests for reprints should be sent to Ronald F. Jarman, Department of Educational Psychology, Faculty of Education, University of British Columbia, Vancouver, British Columbia, Canada V6T $1 \mathrm{W5}$.
1,000 -cps tones with a comparison pattern of tones. All tones are .15-sec in duration. Variations in patterns are created by short pauses of $.35 \mathrm{sec}$ and long pauses of $1.35 \mathrm{sec}$.

\section{Auditory-Visual Matching}

The items in this test utilize an auditory pattern as a stimulus (as in the auditory-auditory test), which is matched with a comparison visual display of dots. For a dot 1 unit in diam, a short gap is .80 units in length, and a long gap is 7.17 units in length.

\section{Visual-Auditory Matching}

This test is the converse of auditory-visual matching, with the same specifications; the visual display is the stimulus portion of each item and the comparison section is a set of tones.

\section{Visual-Visual Matching}

Sets of visual patterns are used with the same specifications as above. The first pattern of each set is compared to the second pattern.

\section{TEST CONSTRUCTION}

In the construction of the auditory portions of the tests, the following equipment is used: (1) Heathkit audio generator Model 1G-72; (2) Akai reel-to-reel tape recorder Model GX 365D; (3) Akai cassette tape recorder Model GXC 38D (with Dolby hiss reduction); and (4) Stoelting universal timing module (timer Model SA600, power unit Model SA590).

$A$ reduction is made in the tape speed and the tone frequency during recording to take advantage of the fact that a 1,000-cps tone can be created by recording a 250 -cps tone at a tape speed of $1-7 / 8 \mathrm{in} . / \mathrm{sec}$, and then playing the tape at a speed of $7-1 / 2 \mathrm{in} . / \mathrm{sec}$. Thus, it is possible to quadruple all of the times used in recording through the use of the slower tape speed. This makes it feasible, albeit demanding in terms of manual coordination, to record all of the item patterns consecutively. A conversion of the specifications for a tape speed of $1-7 / 8 \mathrm{in} . / \mathrm{sec}$ results in the following: 
(1) Frequency of tones, $250 \mathrm{cps}$; (2) length of tones, $.60 \mathrm{sec}$; (3) length of short pauses, $1.40 \mathrm{sec}$; and (4) length of long pauses, $5.40 \mathrm{sec}$.

Bank 1 of the timer is used for closed-circuit timing; Banks 2 and 3 are wired for open-circuit timing such that the lesser of the two times that are specified on these banks is chosen automatically by the timer as the open-circuit period. For example, if Bank 2 is set at $1.40 \mathrm{sec}$ and Bank 3 at $5.40 \mathrm{sec}$, Bank 2 is chosen. However, if Bank 2 is increased by a factor of 10 , giving a setting of $14 \mathrm{sec}$ (easily accomplished by a manual switch on the timer), Bank 3 is chosen for the open circuit.

To construct the tapes, the reel-to-reel recorder is set at a speed of 1-7/8 in./sec recording on Track 1 only. The audio generator is set at $250 \mathrm{cps}$ (constant tone) and the timing module is placed between the recorder and the audio generator as a circuit switcher, using the following settings: Bank 1, .60 sec; Bank 2, $1.40 \mathrm{sec}$; Bank 3, 5.40 sec.

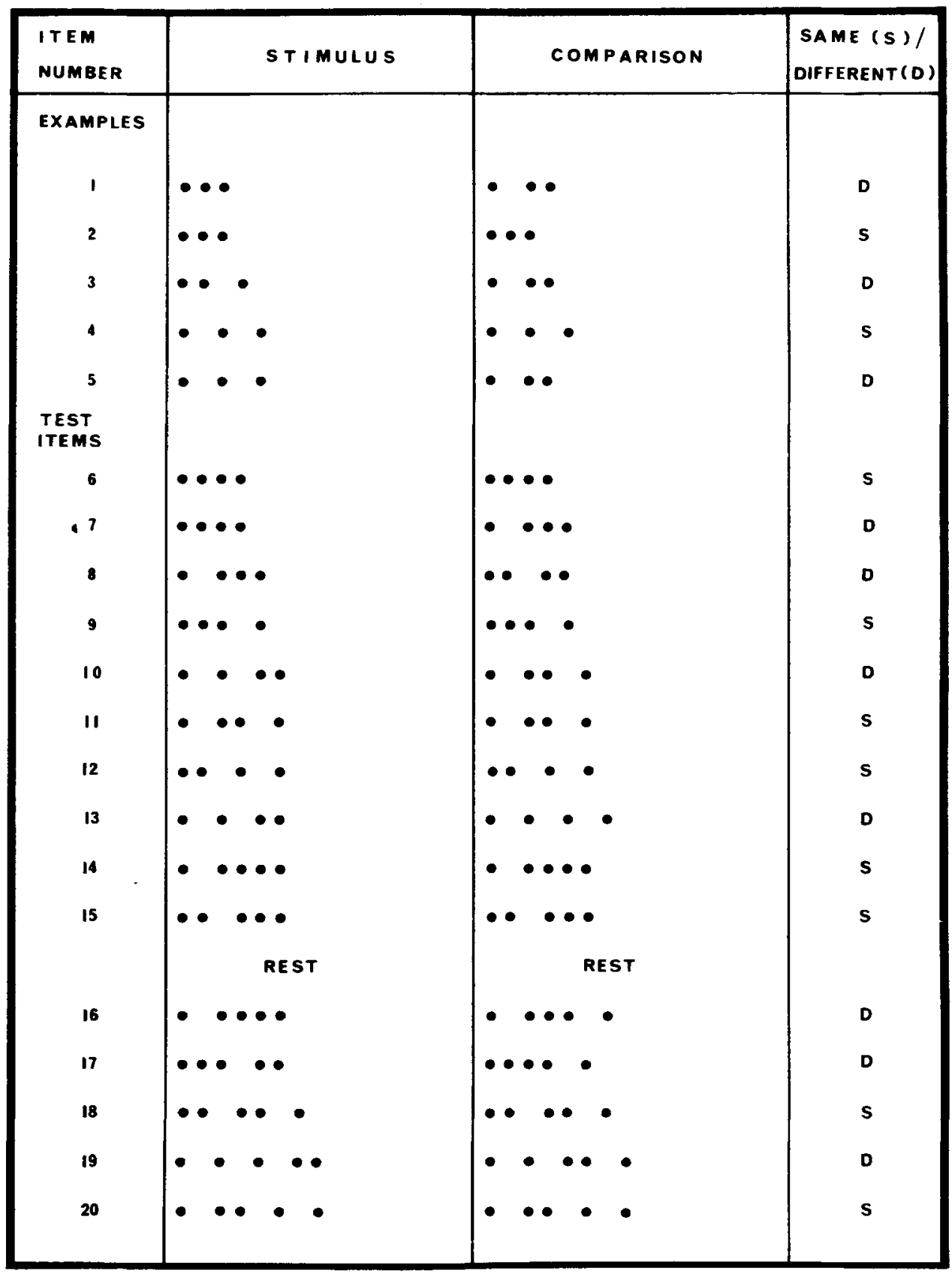

Figure 1. Item patterns for auditory and visual cross-modal and intramodal matching tests. 
Figure 1 (Continued)

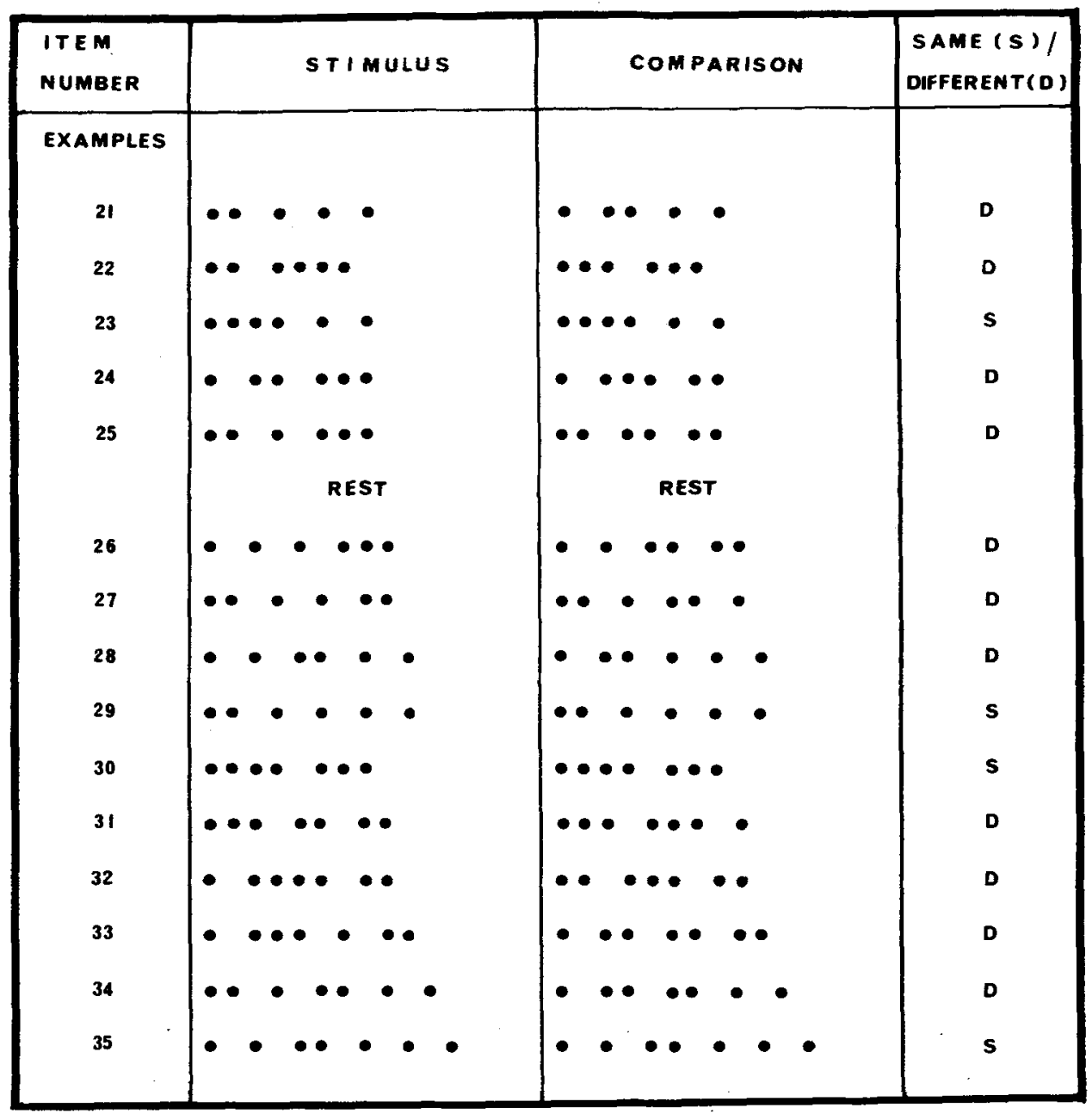

For each item in turn, the following sequence occurs: During the $1.40-\mathrm{sec}$ interval immediately prior to the first tone of the stimulus portion of the item, the volume of the audio generator is set at medium. Unless changed, tones of $.60-\mathrm{sec}$ duration, with pauses of $1.40 \mathrm{sec}$, are recorded. This procedure is modified at each point in the stimulus patterns for which a long pause is specified. During the $.60 \mathrm{sec}$ of tone recording immediately prior to the long pause, the timing setting of Bank 2 is increased manually by a factor of 10 , setting Bank 2 at 14 . The result is that the timer selects Bank 3 for an open circuit, providing a pause period of $5.40 \mathrm{sec}$ following the tone. By switching the setting on Bank 2 manually in this fashion, pauses of $1.40 \mathrm{sec}$ and $5.40 \mathrm{sec}$ are generated according to the design of the item patterns.

In order to create an interval between the stimulus and comparison sections of each item, the audio generator is reset at zero volume at the end of the recording of the last tone in the stimulus section of the item. With the volume off, the timer completes six cycles with the circuit closed for $.60 \mathrm{sec}$ and open for $1.40 \mathrm{sec}$. The comparison portion of the item begins by resetting the volume to medium during the last opencircuit period of the six cycles. The same procedure is used following recording of the comparison portion of the item, in order to create a subject-response interval on the tapes; the audio generator volume is set to 0 while 16 cycles of open and closed circuits are completed.

Thus, a continuous recording is made of the complete auditory-auditory matching test-item patterns, as specified in Figure 1. A script and directions for each item are then recorded such that they mesh with the tone patterns, using a sound-on-sound procedure. The reel-to-reel recorder is set at a speed of $7-1 / 2 \mathrm{in} . / \mathrm{sec}$, with Track 1 set for listening and Track 2 set for recording. The script for auditory-auditory matching is recorded on Track 2 and, then, while listening to the tones of each item in turn, a number designation for the item is recorded, followed by the cue word "Ready." After the stimulus tones, the word "and" is recorded 


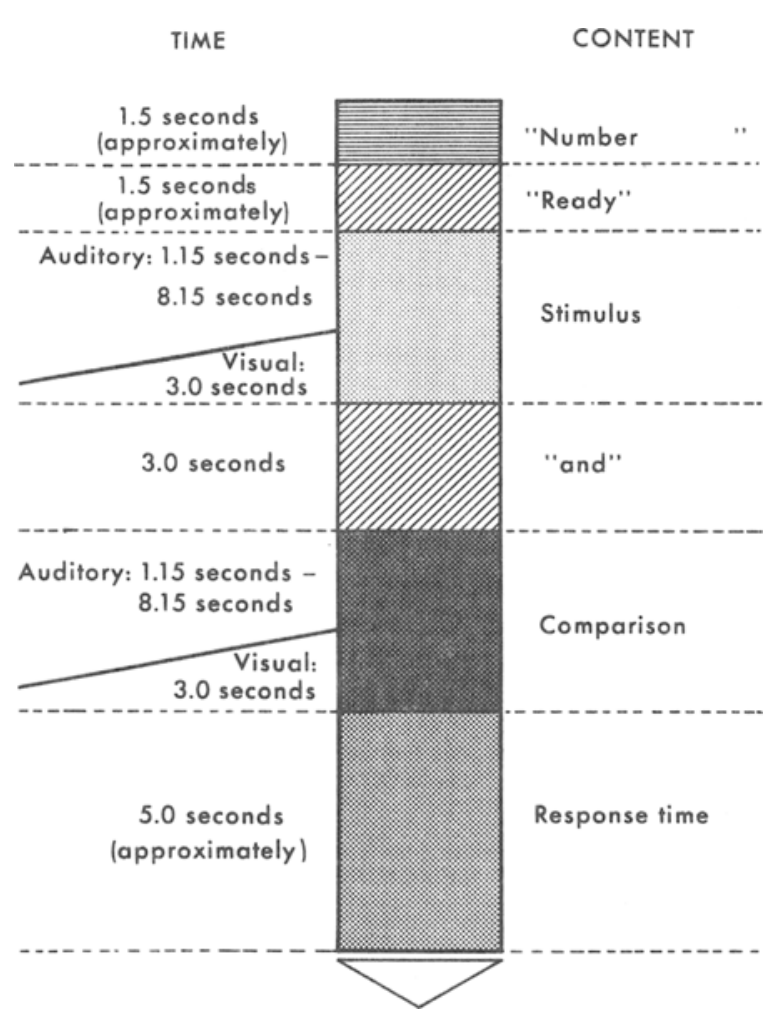

Figure 2. Structure of items.

to designate the separation between the stimulus and comparison patterns. Using this timing, each item structure is established as shown in Figure 2.

Once the complete auditory-auditory matching tape is recorded on both tracks, the tracks are played simultaneously and the recording is switched to a single track on a cassette tape using the Akai portable recorder.

Using the master auditory-auditory tape, tapes for auditory-visual, visual-auditory, and visual-visual matching can be constructed. The scripts for each of the tests are recorded on separate tapes and then portions of the auditory-auditory tape are transferred to new tapes, dependent upon which section of the item in a test is to be visual. In recording the auditory. visual matching tape, for example, the auditory-auditory matching tape is recorded with deletion of the comparison section of the items. This creates a timed blank section on the tape, during which the visual portion of the item can be shown. Cues for activating the slide projector are then placed on the tape in order to supply timing for the display of slides. The duration for displaying the slides may be varied according to the complexity of the patterns or held constant as in the tests described here.

\section{REFERENCES}

Chalfant, J. C., \& Scheffelin, M. A. Central processing dysfunction in children: $A$ review of research, NINDS, No. 9, Washington, D.C: U.S. Department of Health, Education and Welfare, 1969.

FreIDEs, D. Human information processing and sensory modality: Cross-modal functions, information complexity, memory, and deficit. Psychological Bulletin, 1974, 81, 284-310.

\section{NOTE}

1. The rationale for the design of the patterns is not given here. Information regarding pattern design and the psychometric characteristics of the tests can be obtained from the author upon request. Also available is a description of a simple technique for constructing the visual portions of the tests. 